\title{
A Probable New Globular Cluster in the Galactic Disk
}

\author{
Jay Strader ${ }^{1,2}$, Henry A. Kobulnicky ${ }^{3}$ \\ jstrader@cfa.harvard.edu, chipk@uwyo.edu
}

\begin{abstract}
We report the discovery of a probable new globular cluster in the disk of the Milky Way. Visible in 2MASS and the GLIMPSE survey, it has an estimated foreground extinction of $A_{V} \sim 24 \mathrm{mag}$. The absolute magnitude of the cluster and the luminosity function of the red giant branch are most consistent with that of an old globular cluster with a mass of a few $\times 10^{5} M_{\odot}$ at a distance of $4-8 \mathrm{kpc}$.
\end{abstract}

Subject headings: globular clusters: general — galaxies: star clusters

\section{Introduction}

Harris (2001) estimated that there were 20 unknown Galactic globular clusters hidden behind substantial foreground extinction in the disk or behind the bulge. Subsequent near-IR surveys of the disk have borne out the prediction of missing clusters, two of which have been discovered in 2MASS by Hurt et al. (2000). Another cluster, GLIMPSE-C01, was found by Kobulnicky et al. (2005) using the Spitzer/IRAC GLIMPSE survey (Benjamin et al. 2003) of the Galactic plane. The importance of the Galactic globular cluster system in understanding the formation, evolution, and destruction of globular clusters motivates continuing efforts to finish the census of clusters.

In this paper we report the discovery of a probable globular cluster at Galactic coordinates $l=14.13, b=-0.64\left(\mathrm{~J} 2000\right.$ coordinates: $\left.18^{\mathrm{h}_{1}} 18^{\mathrm{m}_{30} \mathrm{~s}}-16^{\circ} 58^{\prime} 36^{\prime \prime}\right)$. This object was identified by Mercer et al. (2005) in a search for star clusters in GLIMPSE, although it was not suggested to be a globular cluster. As this cluster is \#3 in their catalog, we refer to the object as Mercer 3. This is relatively consistent with the rather confused naming conventions for Galactic globular clusters.

\footnotetext{
${ }^{1}$ Harvard-Smithsonian Center for Astrophysics, Cambridge, MA 02138

${ }^{2}$ Hubble Fellow

${ }^{3}$ Department of Physics \& Astronomy, University of Wyoming, 1000 E. University, Laramie, WY 82071
} 


\section{Imaging}

Figure 1 and 2 show 2MASS $J H K$ images of a $2.4^{\prime} \times 2.4^{\prime}$ area around Mercer 3 , as well as a red DSS image on the same scale. The cluster is clearly visible in $H$ and $K$ but disappears in $J$, indicative of the high foreground reddening. There is no evidence of the cluster in the DSS image. In Figure 3 a composite color image of IRAC Band $2(4.5 \mu \mathrm{m})$, Band $4(8 \mu \mathrm{m})$, and MIPS $24 \mu \mathrm{m}$ is shown. The morphology of the cluster is similar in the IRAC bands to that in $H$ and $K$. Patchy, diffuse emission is visible across the frame. However, there is no evidence of a bubble or shell that could suggest a young cluster.

An elongated, opaque cloud dominates the composite image, located only $3^{\prime}$ in projection from the cluster. Smaller dark clouds are located across the frame. We hypothesize that this large cloud complex is located in the foreground of the cluster and is the primary cause of the large extinction we derive later in the paper.

Using the 2MASS images, we performed integrated aperture photometry within a radius of $75^{\prime \prime}$ centered on the cluster, using a concentric sky aperture between 75 and 90". We do not claim that $75^{\prime \prime}$ is certain to be the edge of the cluster in a meaningful sense, but the extinction becomes noticeably variable at larger radii and so a larger aperture cannot be used. Thus light in the outermost parts of the clusters will be lost. A competing effect is that our sky aperture may have larger extinction than the inner parts of the cluster, leading to an undersubtraction of the background and so an overestimate of the cluster luminosity. It is difficult to assess the relative importance of these two effects and we caution the reader that our total magnitudes are likely to be uncertain at least at the $0.2-0.3$ mag level.

We obtain total integrated magnitudes of $H=7.3$ and $K=6.1$. The half-light radius of the cluster in $K$ is $\sim 39^{\prime \prime}$, but this is a lower limit due to the uncertainty in the amount of light at large radii and the extinction gradient in the image. For the likely distance range derived in $\S 4,4-8 \mathrm{kpc}$, this corresponds to a half-light radius between 0.8 and $1.5 \mathrm{pc}$. The low end of the range is smaller than nearly all Galactic GCs, while a value of 1.5 pc is smaller than typical but not unusual. It is noteworthy that GLIMPSE-C01 and another recent Galactic plane discovery FSR 1767 (Bonnato et al. 2007) also have very small halflight radii of $\sim 0.6-0.7$ pc (Kobulnicky et al. 2005; Bonatto \& Bica 2008). It is unclear at present whether these small radii are accurate or are an artifact of the high extinction and the resultant difficulty in obtaining good surface brightness profiles. 


\section{Stellar Photometry}

We use point source photometry taken from the Version 2.0 Data Release of GLIMPSE and matched with 2MASS sources. Within a radius of $30^{\prime \prime}$ from the cluster center, we select only those sources that are detected in all of $H, K$, and IRAC Bands 1 and 2. 25 stars fit these criteria. Figure 4 shows $K$ vs. $H-K$ and $K$ vs. $K-3.6$ color-magnitude diagrams as observed; no extinction corrections have been applied. $12 \mathrm{Gyr}[Z / \mathrm{H}]=-2$ isochrones from Marigo et al. (2008) are plotted, assuming a distance of $5 \mathrm{kpc}$ and $E(B-V)=7.7$ (the values derived in $\S 3.1$ and $\S 4)$.

Both panels show a broad column of stars extending across $\sim 3$ mag in $K$. The cutoff at $K \sim 14$ is due to the photometric limit of 2MASS; the IRAC images go somewhat deeper. A reasonable assumption is that these stars are the brightest red giants in the cluster, and that the spread in colors is due to differential extinction.

\subsection{Extinction}

We can constrain the extinction toward the cluster by noting the remarkable fact that the IR colors of red giants vary little with age or metallicity, except at the tip of the red giant branch where there are few stars. The typical spread in colors is no more than $\sim 0.1$ mag in $H-K$ and $0.05 \mathrm{mag}$ in $K-3.6$. Thus we can use a color-color plot to estimate the extinction with no knowledge of the metallicity or age of the cluster.

Figure 5 is an $K-3.6$ vs. $H-K$ color-color plot. Overplotted are lines representing the reddened mean color of the upper red giant branch for a 12 Gyr old globular cluster; the isochrones used are Marigo et al. (2008). Two extreme metallicities are plotted, -2 and 0 . The reddenings range from $E(B-V)=6$ to 9 , with crosses marking each magnitude of reddening. This figure shows that the unknown metallicity of the cluster has a minor effect on the red giant branch color compared to the spread in the points, suggesting that the differential extinction dominates the error. Since the lines do not pass directly through the center of points, one derives a different reddening from each of the colors. In $H-K$ the mean reddening appears to be $E(B-V) \sim 7.8$, compared to $E(B-V) \sim 7.5$ for $K-3.6$. The differential reddening is at least 1 mag in both colors, though this may be exaggerated by the contamination of our sample with field stars (the stars lying far from the central clump in Figure 5, for example, are unlikely to be cluster members). Stellar population models are better tested in the classic near-IR bands of $H$ and $K$ than in the newer Spitzer bands, so we tend to slightly favor the $H-K$ value. Thus we will adopt $E(B-V) \sim 7.7$ as our fiducial mean reddening, keeping in mind the presence of large differential reddening. 
An $E(B-V)$ value of $\sim 7.7$ is extraordinarily high, corresponding to $A_{K} \sim 2.8$ and $A_{V} \sim 24$. We can do a sanity check by noting that the cluster is not detected in the 2MASS $J$ image. The brightest red giants have $K \sim 11$, equivalent to an extincted $J \sim 16$. Below $J \sim 16$, especially in crowded regions, the completeness of 2MASS drops significantly, consistent with the absence of anything but a few stars at the position of the cluster in the $J$ image in Figure 1. However, if the reddening were as low as $E(B-V)=5.5$ or 6 , then the brighter red giants would be visible in $J$. We conclude that our derived reddening is consistent with the lack of the cluster in the 2MASS $J$ image.

\subsection{The Source of the Extinction}

As discussed above, Mercer 3 is located several arcmin in projection from a large IR dark cloud. It is possible that this cloud is associated with the material responsible for the large reddening towards the star cluster.

In their discovery paper of a globular cluster in GLIMPSE, Kobulnicky et al. (2005) used relatively high resolution CO data from the Galactic Ring Survey (Jackson et al. 2006) as a consistency check on the extinction toward their cluster. Unfortunately, Mercer 3 falls outside of the footprint of this survey, so we must fall back on older, lower-resolution data from the Massachusetts-Stony Brook Galactic Plane CO Survey (Clemens et al. 1986). The

resolution of these data is $\sim 6^{\prime}$, too low to compare the morphology of the cloud in Figure 3 to the $\mathrm{CO}$ maps.

We downloaded a data cube from this survey covering the position of our cluster and extracted an integrated $\mathrm{CO}$ spectrum at the position of our cluster. The only significant feature is a strong peak at $20 \mathrm{~km} / \mathrm{s}$. Integrating over the profile gives an intensity $I_{C O}=93$ $\mathrm{K} \mathrm{km} / \mathrm{s}$. This may be converted into an $\mathrm{H}_{2}$ column density and optical extinction using the equations in Bohlin et al. (1978; see also Kobulnicky \& Skillman 2008): $N_{H_{2}}=3 \times 10^{20} I_{C O}$ and

$$
A_{V}=3.1 \frac{2 N_{H_{2}}}{5.8 \times 10^{21}}
$$

Substitution yields $A_{V} \sim 30$ along this line of sight, generally consistent with the value derived from the color-color diagram. These equations assume that the CO is not optically thick and that there is no contribution of $\mathrm{H}$ I to the extinction, and so represent a lower limit. On the other hand, some of the molecular gas may be behind the cluster; due to the low resolution of the data, the gas might also be associated with a different cloud that is in 
front of the cluster but not contributing to the foreground extinction.

If we assume that the CO cloud is predominately in the foreground, then we can use its velocity to constrain the near/far distance of the cluster. For $v=20 \mathrm{~km} / \mathrm{s}$ and a Galactic $l=14.1$, the near/far distances are 2.4 and $14.1 \mathrm{kpc}$. We can then, at the very least, take $2.4 \mathrm{kpc}$ as a lower limit on the cluster distance. In the next section we will use the color-magnitude diagram of cluster stars to derive an upper limit on the distance.

\section{Luminosity Function, Age, and Distance}

An additional constraint on the distance and age of the cluster comes from the stellar luminosity function (LF). With only 25 stars in the complete sample, creating a useful $K$ band LF is impractical. However, the GLIMPSE data are deeper, so if we relax the restriction on matches with 2MASS, we can select a sample of stars with detections in IRAC bands 1 and 2. Within 30 "of the cluster center, there are 70 such stars. Figure 6 shows the $3.6 \mu \mathrm{m}$ LF plotted as a density estimate, using an Epanechnikov kernel and a bin width of 0.25 mag. The main features of the LF are: (i) a lack of stars brighter than $m_{3.6}=9$, (ii) significant incompleteness below $m_{3.6} \sim 13$, and (iii) a gently upward sloping LF between these two limits. Overplotted are theoretical LFs from Marigo et al. (2008) for solar metallicity and a range of ages from 1 Gyr to 12 Gyr (for old ages, the differences between metal-rich and metal-poor LFs in $3.6 \mu \mathrm{m}$ are small compared to the quality of our data and the effect of differential reddening). These have been scaled in distance and normalization to produce the best match for each age.

A generic feature of the LFs for ages younger than $\sim 2-3$ Gyr is a bump in the LF at the brightest magnitudes due to red supergiants. This bump is especially pronounced for ages of $\sim 1$ Gyr and for certain younger ages. No such feature is seen in the observed LF. Thus, independent of the cluster distance, the LF is inconsistent with Mercer 3 being a young star cluster. The LF is most consistent with that of a relatively old open or globular cluster.

Further constraints on the distance of the cluster come from the assumption of a particular age. For an 12 Gyr solar metallicity stellar population, the maximum distance comes from identifying the brightest stars with the tip of the giant branch. This corresponds to an extincted distance modulus of $m-M \sim 16$, or $m-M_{0} \sim 14.4$ using $A_{3.6}=1.6$ (assuming $E(B-V)=7.7)$. Thus the maximal cluster distance is $\sim 7.6 \mathrm{kpc}$. The shape and normalization of the LF appear to be somewhat better fit by a distance of $5.0 \mathrm{kpc}$ (this is the fit

plotted in Figure 6), although the fit is far from perfect. Distances of $4 \mathrm{kpc}$ or smaller are 
poor fits, as the theoretical LFs begin to rise steeply in a way unmatched by the data. This might partially be addressed by positing incompleteness at a brighter magnitude. Recall that the near distance limit from the CO data was $2.4 \mathrm{kpc}$. We conclude that a plausible distance range for an old cluster is $4-8 \mathrm{kpc}$, with a value closer to the middle of that range somewhat favored.

Assuming an age near the opposite extreme of the allowed range gives an upper limit on the distance. As shown in Figure 6, a 5 Gyr solar metallicity population appears to fit about as well as did the 12 Gyr LF. The implied distance is $\sim 12-13 \mathrm{kpc}$ (with a large error). What does this long distance imply for the mass of the cluster? Given the extinction and total $K$ magnitude discussed earlier, we derive $M_{K} \sim-12.1$ for a distance of $12 \mathrm{kpc}$. Using Maraston (2005) models with a Kroupa initial mass function, this is equivalent to a mass of $\sim 8 \times 10^{5} M_{\odot}$. Mercer 3 would be one of the most massive star clusters in the Galaxy.

Alternatively, if we assume a distance of $5 \mathrm{kpc}$ and an age of $12 \mathrm{Gyr}$, the implied mass is $\sim 2-3 \times 10^{5} M_{\odot}$ (depending on metallicity). This is close to the peak of the lognormal globular cluster luminosity function and would essentially peg Mercer 3 as a typical Milky Way globular cluster - keeping in mind that the total cluster luminosity is still quite uncertain.

As a conservative check on this mass estimate, we set aside the integrated $K$-band magnitude of the cluster for a moment and simply coadd the flux from all of the sources that lie along the red giant branch in Figure 4 . This gives $K \sim 8.5$. We then make the almost absurd assumption that we have detected all of the red giants in the cluster (unlikely both because of incompleteness and because we are only considering sources within $30^{\prime \prime}$ of the center). Noting that standard stellar population models (e.g., Worthey 1994) predict that $50 \%-60 \%$ of the total $K$-band flux of an intermediate-age to old object will be from the red giant branch, we derive a total $K \mathrm{mag}$ of $\sim 7.8$. For an old object at $5 \mathrm{kpc}$, this corresponds to a mass of $\sim 5 \times 10^{4} M_{\odot}$-less massive than a typical globular cluster, but not unusual, and still much more massive than nearly all open clusters.

Our conclusion from this line of argument is that Mercer 3 is most likely to be a typical old globular cluster, but we cannot rule out a less massive globular cluster or a more massive intermediate-age object.

\section{Discussion}

A secure identification of Mercer 3 as an old globular cluster will require moderately deep near-IR photometry. The predicted main sequence turnoff is at $K \sim 19$; if instead 
it is a massive intermediate-age cluster, the turnoff will be more than a magnitude fainter, and the shape of the subgiant branch will be significantly different. Due to the large and differential reddening, an accurate estimate of the cluster metallicity will probably require near-IR spectroscopy. This is easily accomplished as the brightest red giants have $K \sim 11$.

Mercer 3 is the second probable globular cluster discovered using Spitzer and 2MASS; a further two globular clusters were found using 2MASS alone. At the opposite end of parameter space, Koposov et al. (2007) discovered two extraordinarily low-mass globular clusters in the Sloan Digital Sky Survey at heliocentric distances of $\sim 40-50 \mathrm{kpc}$. The continuing pace of these discoveries suggests that the Galactic cluster census is far from complete.

J. S. was supported by NASA through a Hubble Fellowship, administered by STScI. We thank Dan Clemens, Laura Chomiuk, and Beth Willman for useful comments on the manuscript.

\section{REFERENCES}

Benjamin, R. A., et al. 2003, PASP, 115, 953

Bohlin, R. C., Savage, B. D., \& Drake, J. F. 1978, ApJ, 224, 132

Bonatto, C., \& Bica, E. 2008, A\&A, 479, 741

Bonatto, C., Bica, E., Ortolani, S., \& Barbuy, B. 2007, MNRAS, 381, L45

Clemens, D. P., Sanders, D. B., Scoville, N. Z., \& Solomon, P. M. 1986, ApJS, 60, 297

Harris, W. E. 2001, Saas-Fee Advanced Course 28: Star Clusters, 223

Hurt, R. L., Jarrett, T. H., Kirkpatrick, J. D., Cutri, R. M., Schneider, S. E., Skrutskie, M., \& van Driel, W. 2000, AJ, 120, 1876

Jackson, J. M., et al. 2006, ApJS, 163, 145

Kobulnicky, H. A., \& Skillman, E. D. 2008, AJ, 135, 527

Kobulnicky, H. A., et al. 2005, AJ, 129, 239

Koposov, S., et al. 2007, ApJ, 669, 337

Maraston, C. 2005, MNRAS, 362, 799 
Marigo, P., Girardi, L., Bressan, A., Groenewegen, M. A. T., Silva, L., \& Granato, G. L. 2008, A\&A, 482, 883

Mercer, E. P., et al. 2005, ApJ, 635, 560

Worthey, G. 1994, ApJS, 95, 107 

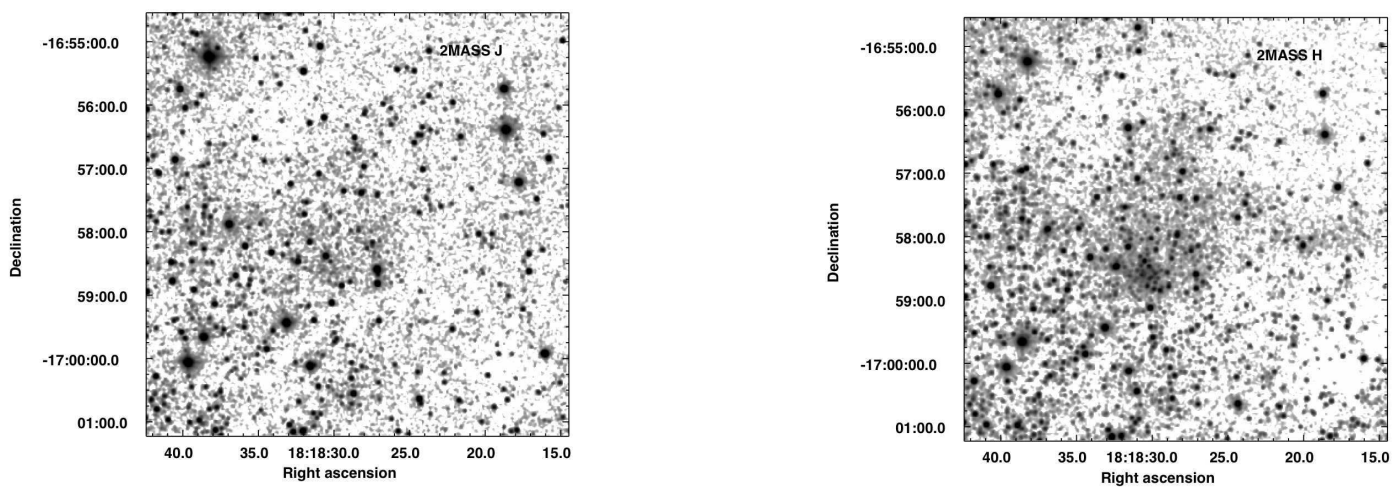

Fig. 1. - Postage stamp images of the cluster in 2MASS $J H$. The cluster is not visible in the $J$ image due to high extinction.
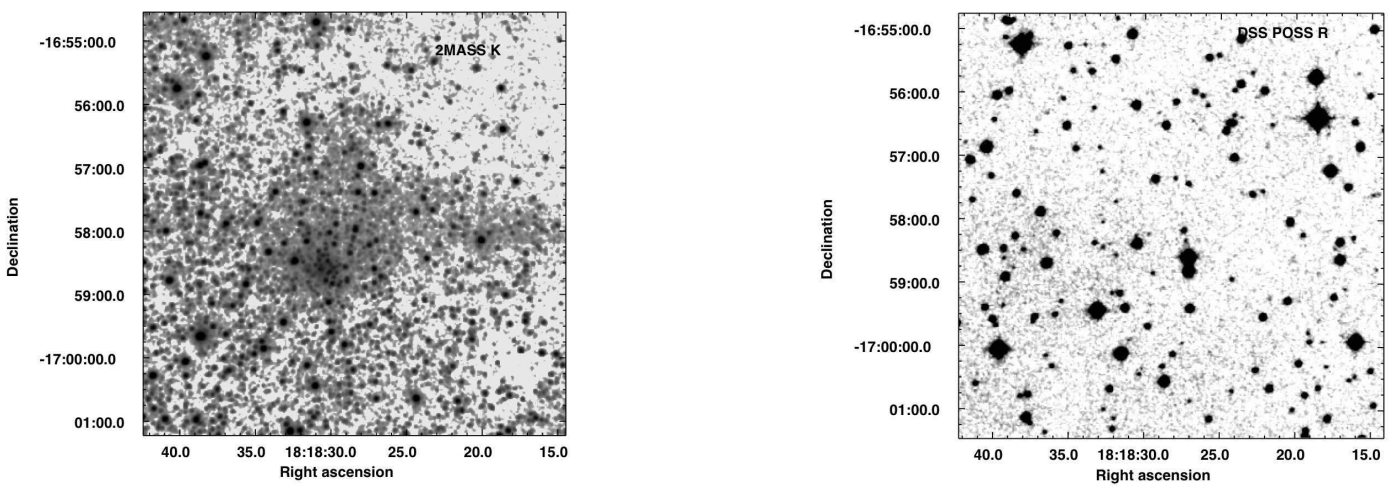

Fig. 2.- Postage stamp images of the cluster in 2MASS $K$ and in red DSS. The cluster is not visible in DSS due to high extinction. 


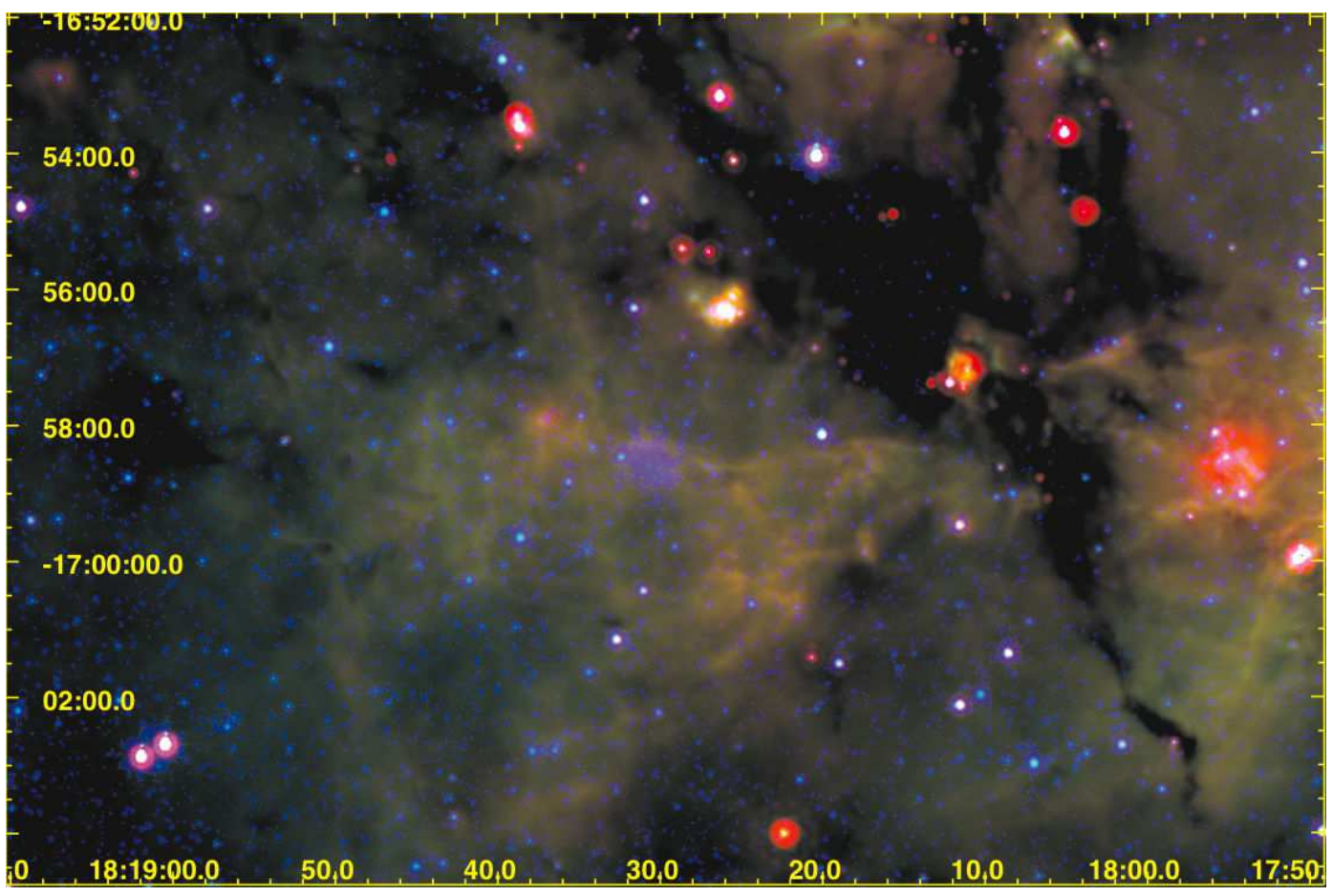

Fig. 3.- Three-color image using IRAC Bands 2 and 4 and MIPS $24 \mu \mathrm{m}$, showing the cluster in the center of the image and its environment. Patchy extinction is present throughout the image, and a large infrared dark cloud is present only a few arcmin in projection from the cluster. This cloud may be associated with high extinction toward the cluster. 

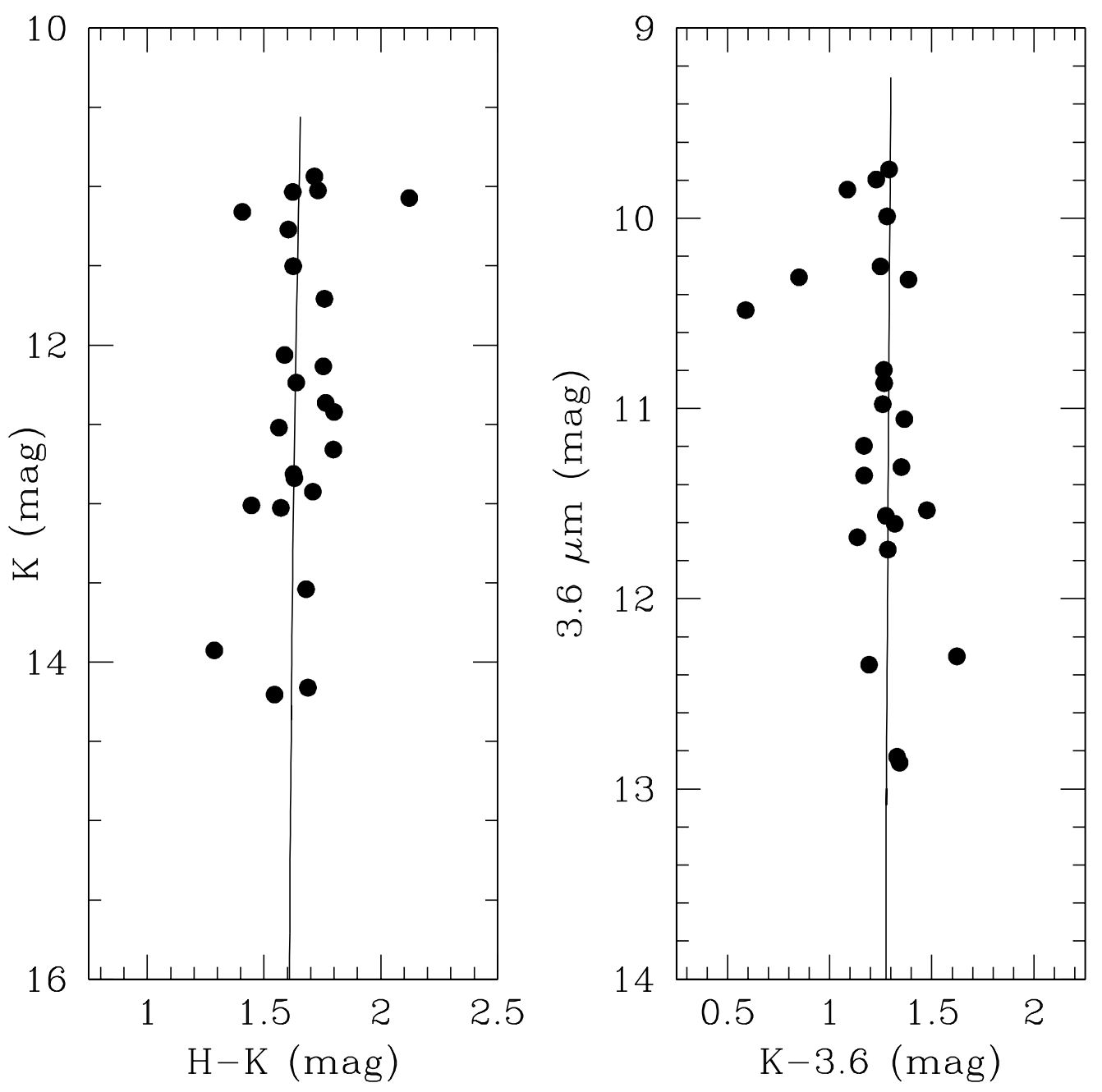

Fig. 4. $-K$ vs. $H-K$ and 3.6 vs. $K-3.6$ color-magnitude diagrams of Mercer 3. 12 Gyr, $[Z / \mathrm{H}]=-2$ isochrones from Marigo et al. (2008) assuming $E(B-V)=7.7$ and a distance of $5 \mathrm{kpc}$ are overplotted. 


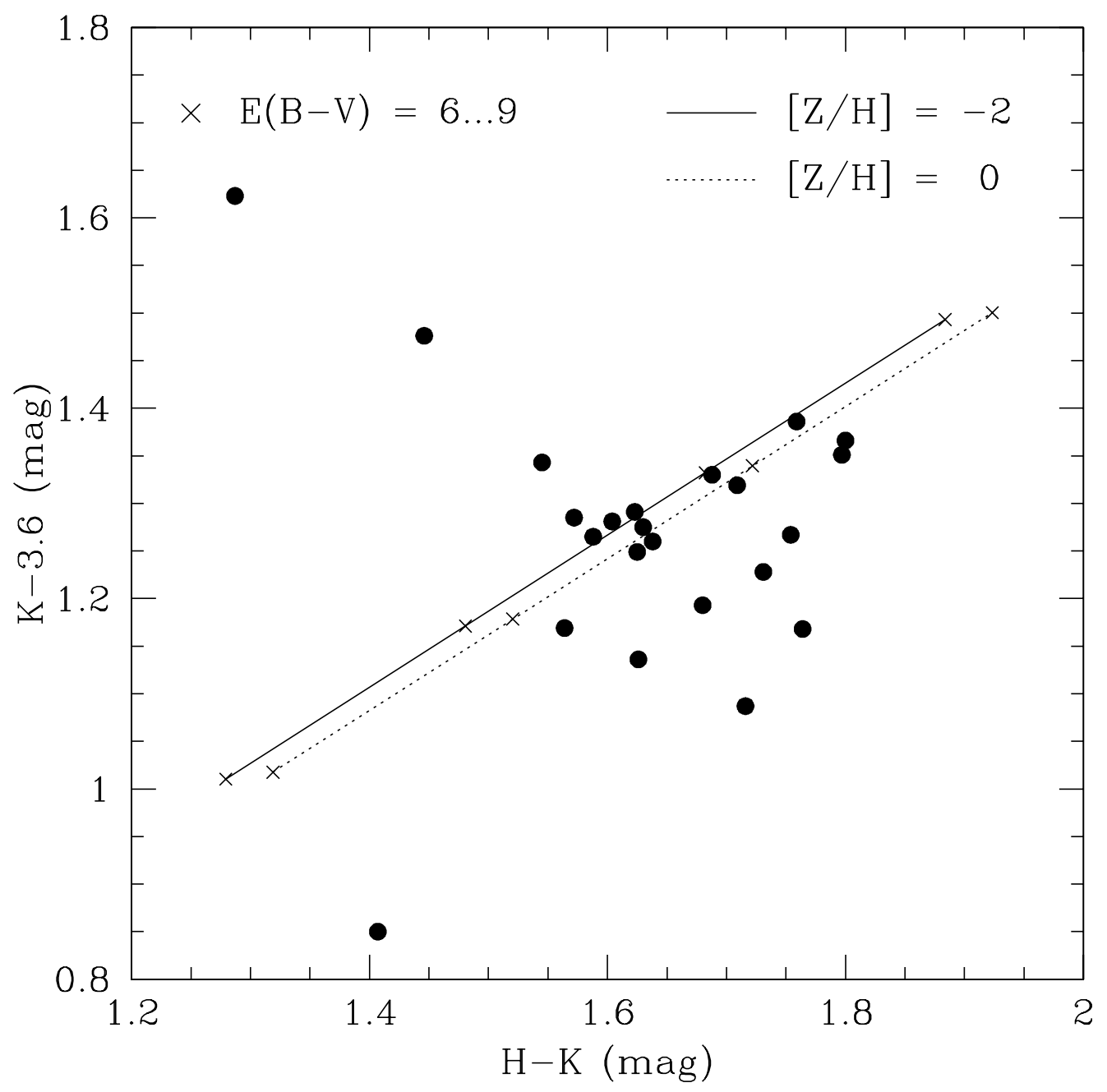

Fig. 5.- $K-3.6$ vs. $H-K$ color-color diagram of red giants in the cluster. Mean colors for the upper red giant branch using $12 \mathrm{Gyr}$ isochrones with $[Z / \mathrm{H}]=-2$ (solid line) and 0 (dotted line) are overplotted; $E(B-V)$ ranges from 6 to 9 with crosses marking magnitude intervals. A value in the interval $7.5 \lesssim E(B-V) \lesssim 7.8$ is favored. 


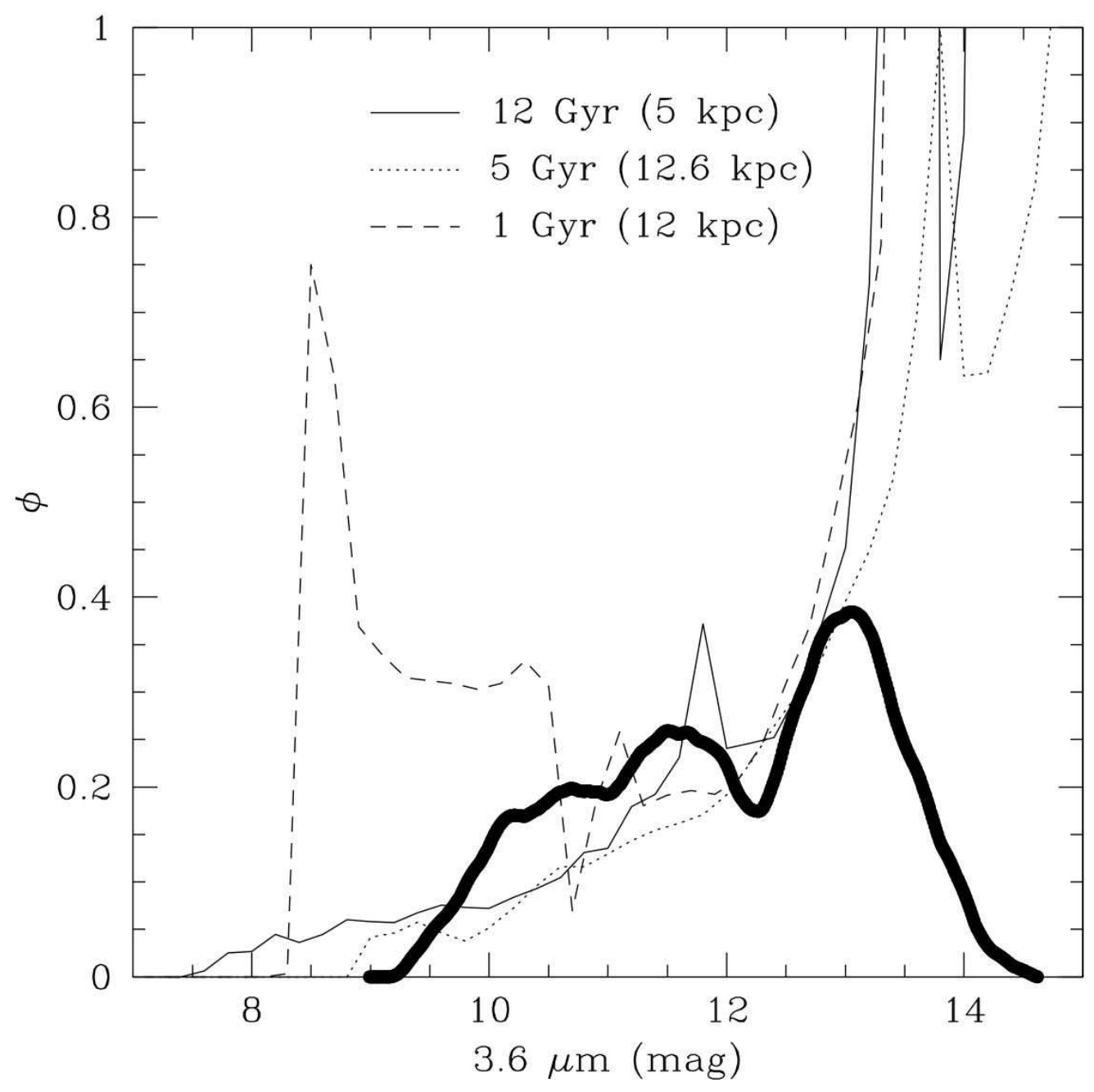

Fig. 6.- Observed $3.6 \mu \mathrm{m}$ luminosity function compared to theoretical luminosity functions of solar metallicity from Marigo et al. (2008). These have ages of 12 Gyr (solid), 5 Gyr (dotted), and 1 Gyr (short dashed). Ages younger than a few Gyr are disfavored because of the lack of red supergiants. 